SCIENTIFIC REPORT

\title{
Further validation of the Daily Living Tasks Dependent on Vision: identification of domains
}

\author{
P M Hart, M R Stevenson, A-M Montgomery, K A Muldrew, U Chakravarthy
}

Br J Ophthalmol 2005;89:1127-1130. doi: 10.1136/bjo.2004.059683

Aim: To examine the Daily Living Tasks Dependent on Vision (DLTV), a visual function questionnaire for domain structure, and redundancy.

Method: 235 subjects underwent full ophthalmic assessment and completed the DLTV questionnaire by interview. Principal component analysis with varimax rotation and item response theory (IRT) were used to assign the items to domains. The internal consistency of each domain was examined using Cronbach's alpha. Redundancy was assessed by regressing each item in a domain against the remainder of items in that domain.

Results: Four domains were identified. Domain 1 was formed by nine items, which after applying IRT were seen to be among the most difficult questions in the instrument. Domain 2 contained eight items, all of which fell in the easier half of the instrument on applying IRT. Domain 3 contained only three items, all of which were among the easier questions and appear to deal with peripheral vision function. Domain 4 consisted of two items dealing with adaptation to light and dark conditions. Cronbach's alpha for each domain was $0.96,0.93,0.73,0.66$. Redundancy was found to be present in domain 1, which was therefore reduced by two items, with little effect on internal consistency.

Conclusions: The authors believe using the domains identified in this report will optimise the information provided by patients on their ability to function on visually demanding tasks.

$\mathrm{T}$ he Daily Living Tasks Dependent on Vision questionnaire (DLTV) was developed in 1995 specifically for use in older people with age related macular degeneration (AMD). ${ }^{12}$ It formed part of the assessment of patients participating in a randomised controlled clinical trial of external beam radiotherapy in subfoveal choroidal neovascularisation, the primary outcomes of which have already been reported. ${ }^{3}$

In our previous validation studies we have shown that combinations of conventional measures of vision in the better eye were better than single measures in predicting responses to items within the instrument. ${ }^{1}{ }^{2}$ We have demonstrated discriminant validity, showing that the DLTV could distinguish between patients with cataract and those with AMD.

Principal component analysis (unrotated) suggested that although not quite unidimensional, the DLTV has a single major component within it. ${ }^{1}$ We now have more data from a larger group of patients with AMD and visual losses of varying severity. We therefore thought it was appropriate to re-examine the construct validity of the questionnaire and optimise its domain structure.

\section{METHODS}

Approval was obtained from the local ethics committee before undertaking this research and the studies were carried out in accordance with the tenets of the Declaration of Helsinki on research in human volunteers.

\section{Subjects}

A total of 235 individuals were recruited and gave informed consent. Patient age ranged from 50 to 97 years with a mean of 74 (SD 9); 82 were male and 153 were female. All patients were diagnosed with AMD.

\section{Measures of vision}

Each participant had distance and near visual acuity and contrast sensitivity measured on each eye separately, according to a specified protocol and under standard conditions of illumination, as previously described. ${ }^{3}$

\section{Visual functioning instrument}

The questions within the DLTV were posed to each participant by a trained interviewer who recorded responses on the questionnaire.

In summary, the DLTV consists of a core of 22 individual items each with a four point ordinal response scale. In addition to questions relating to specific tasks, there are questions asking the individual to describe the degree of confidence they have in walking around in familiar and unfamiliar neighbourhoods. One question, which asked about "difficulty in seeing objects off to the side," allowed most subjects with even severe visual loss from AMD to be able to respond positively, and thus served as a truth indicator.

Further to the 22 core items, four other questions were posed. Patients were asked to rate their general health status on a scale of 1 to 10 . They were also asked to rate their overall distance vision, to rate their overall near vision, and to state agreement or disagreement with the statement "I have to be more careful because of my eye condition."

These latter three questions can be considered to be more global statements of self reported visual impairment and we applied different response scales from that used for the core items. They are not task specific and we applied different response scales from that used for the 22 core items. We therefore examined the responses to these three questions separately, and they have not been included in this interrogation of the DLTV looking for domains. Similarly, the question on overall health status has not been included in the present analysis. The relation of the responses to these four additional questions with the other items will form the basis of a separate report.

Abbreviations: $A M D$, age related macular degeneration; DLTV, daily living tasks dependent on vision; IRT, item response theory; PCA, principal component analysis 


\begin{tabular}{|lllllll}
\hline Table 1 & Measures of vision in the better and worse eyes & & \\
\hline & \multicolumn{3}{l}{ Better eye } & & & Worse eye \\
\cline { 2 - 3 } Measures of vision & Mean (SD) & Range & & Mean (SD) & Range \\
\hline Distance visual acuity (logMAR) & $0.53(0.47)$ & $-0.2-1.7$ & & $1.15(0.51)$ & $0.0-1.7$ \\
Near visual acuity (logMAR) & $0.77(0.51)$ & $0.0-1.7$ & & $1.34(0.41)$ & $0.1-1.7$ \\
Contrast sensitivity (log units) & $1.11(0.45)$ & $0.0-1.8$ & & $0.55(0.54)$ & $0.0-1.7$ \\
\hline
\end{tabular}

\section{Statistical methods}

The Statistical Package for Social Sciences (SPSS) version 11 was used to record, retrieve, and analyse data. Construct validity was examined first using principal component analysis (PCA) with varimax rotation to allocate the 22 items to domains. We examined the effect of varying the cutoff level for eigenvalues from 1.2 to 0.8 (the traditional value is 1.0). Taking a slightly lower value allows more components to emerge. An alternative but similar approach would be to predetermine the number of components and examine their content and eigenvalues. ${ }^{4}$

We also examined the responses to items using item response theory (IRT). IRT is a powerful statistical tool that estimates the probability of a positive response on any question as a function of ability to perform the particular task. We used ordinal regression, based on the methodology of McCullagh, ${ }^{5}$ since the responses were on a four point ordinal scale. The procedure is referred to as PLUM. The item effects are presented as parameter estimates for each item and the syntax used in SPSS rates easy tasks with higher positive values. The methodology is comparable to the Rasch analysis of dichotomous data.

Internal consistency within each domain was examined using Cronbach's alpha. Redundant items were identified by regressing each item in any domain against the remainder of items in the domain and excluding those where the other items explained $75 \%$ or more of the variation in response.

\section{Terminology}

Domains can be considered as sets or combinations of items that behave similarly to one another and that behave differently from other sets within a questionnaire. In this paper we have referred to the sets identified by PCA as components or dimensions. These may or may not be identical to the final set of items deemed be a domain. This decision depends on further assessments, in this case application of IRT.

\section{RESULTS}

\section{Measures of vision}

The mean distance and near acuity and contrast sensitivity of both eyes of the cohort are shown in table 1 .

\section{Construct validity}

Construct validity was examined using PCA with varimax rotation. Applying the traditional or default eigenvalue of 1.0, produced a two component solution which explained $66 \%$ of total variation. Altering the cutoff eigenvalue to 1.2 produced a single component which explained $61 \%$ of total variation, while a cut-off value of 0.8 produced four components which explained $75 \%$ of total variation. This four dimension rotated solution distributed the variation as $29 \%, 25 \%, 11 \%$, and $10 \%$.

Column A in table 2 gives the item loading factor when the Eigenvalue was set at 0.8 resulting in four dimensions. Column B shows the item loading factors when the Eigenvalue was set at 1.2 resulting in a one dimension solution. Some items however have low loading factors.
Taking an arbitrary cut-off value for a loading factor of 0.5 , we would lose three items with the lowest loading factors: the items relating to "seeing objects off to the side," "adjusting to brightness after being in the dark," and "adjusting to darkness after being in the light" would be excluded. These items make very poor contributions to the component, and would be better considered as individual items. The item asking about "difficulty seeing objects off to the side" has the lowest loading factor (0.19) - that is, this roughly equates to it contributing less than one fifth of a complete question.

\section{The four component solution}

The model places each item in the component for which the item shows the highest loading factor. The three items that were found to have poor loading factors in the unidimensional solution were distributed in the later dimensions of the four dimensional solution. "Noticing objects off to one side" was placed in the third component and was joined by an item relating to "seeing and using steps" and an item that asks about "confidence in walking around in one's own neighbourhood." "Difficulty adjusting to brightness after darkness" and "difficulty adjusting to darkness after being in the light," were the two items allocated to the fourth component. The remaining 17 items were allocated as nine to the first component and eight to the second.

\section{Item response theory (IRT)}

Column $\mathrm{C}$ of table 2 provides the parameter estimates for each item, from ordinal regression. The items are effectively ranked in terms of difficulty, the less difficult with higher positive numbers. IRT identified the question relating to "seeing objects off to the side" as the easiest item within the DLTV, and "distinguishing a person's features across the street" as the most difficult.

Setting aside the five items allocated by PCA to components 3 and 4, IRT identified the items in component 1 as being the more difficult items and those in component 2 as the easier to answer. IRT therefore supported the distribution of items into the components obtained by PCA. We took the four components selected by PCA to be the domains within the DLTV.

\section{Internal consistency}

Using Cronbach's alpha, internal consistency was found to be $0.96,0.93,0.73,0.66$ for domains $1,2,3$, and 4 .

\section{Redundancy}

Within each domain all items were regressed against the remainder of the items in that domain. Only in domain 1 were there items where the responses could adequately be explained by the other items. For "reading correspondence," $86.4 \%$ of the variation was explained by the other items within domain 1; and for "identifying money," $77.7 \%$. This finding, together with the high internal consistency of the domain, means that these two items can be considered as 
Table 2 Columns A and B, loading factors for items. (A) When four dimensions are seen in PCA; (B) when one dimension is seen in PCA. The highest loading factor for each item is in bold text. (C) Parameter estimates from ordinal regression, PLUM analysis

\begin{tabular}{|c|c|c|c|c|c|c|}
\hline \multirow[b]{2}{*}{ Items } & \multicolumn{4}{|c|}{ A Loading factors for items } & \multirow{2}{*}{$\begin{array}{l}\text { B Loading } \\
\text { factors for items } \\
\text { Single } \\
\text { dimension }\end{array}$} & \multirow{2}{*}{$\begin{array}{l}\text { C } \\
\text { Parameter } \\
\text { estimates }\end{array}$} \\
\hline & $\operatorname{Dim} 1$ & $\operatorname{Dim} 2$ & $\operatorname{Dim} 3$ & $\operatorname{Dim} 4$ & & \\
\hline Noticing objects off to either side & 0.06 & 0.23 & 0.81 & 0.01 & 0.19 & 4.33 \\
\hline Recognising seasonal changes in the garden & 0.20 & 0.69 & 0.36 & 0.18 & 0.52 & 3.96 \\
\hline Cutting up food on your plate & 0.26 & 0.74 & 0.32 & 0.17 & 0.60 & 3.60 \\
\hline Enjoying the scenery if out for a drive & 0.31 & 0.66 & 0.34 & 0.25 & 0.62 & 3.34 \\
\hline Pouring yourself a drink & 0.38 & 0.61 & 0.35 & 0.24 & 0.65 & 2.75 \\
\hline Using kitchen appliances & 0.50 & 0.55 & 0.29 & 0.29 & 0.73 & 2.69 \\
\hline Seeing steps and using them & 0.45 & 0.34 & 0.50 & 0.30 & 0.59 & 2.66 \\
\hline Reading newspaper headlines & 0.47 & 0.74 & 0.06 & 0.08 & 0.64 & 2.63 \\
\hline Distinguishing a person's features at arm's length & 0.50 & 0.63 & 0.02 & 0.21 & 0.61 & 2.50 \\
\hline Do you feel confident to walk around your own neighbourhood? & 0.47 & 0.21 & 0.62 & 0.30 & 0.54 & 2.42 \\
\hline Cutting your finger nails? & 0.41 & 0.60 & 0.19 & 0.28 & 0.61 & 2.14 \\
\hline Difficulty adjusting to brightness after the dark? & 0.17 & 0.16 & 0.16 & 0.84 & 0.28 & 2.06 \\
\hline Signing documents - eg, cheques & 0.63 & 0.58 & 0.14 & 0.19 & 0.77 & 1.98 \\
\hline Identifying money from a wallet* & 0.69 & 0.53 & 0.19 & 0.16 & 0.79 & 1.94 \\
\hline Difficulty adjusting to darkness after light? & 0.34 & 0.31 & 0.06 & 0.69 & 0.42 & 1.89 \\
\hline Watching TV programmes & 0.61 & 0.44 & 0.29 & 0.24 & 0.71 & 1.62 \\
\hline Reading road signs/street names & 0.62 & 0.48 & 0.20 & 0.28 & 0.73 & 1.06 \\
\hline Reading correspondence* & 0.77 & 0.51 & 0.12 & 0.12 & 0.80 & 0.60 \\
\hline Distinguishing a person's features across the room & 0.71 & 0.37 & 0.14 & 0.29 & 0.69 & 0.42 \\
\hline Feel confident to walk around unfamiliar neighbourhood & 0.72 & 0.23 & 0.39 & 0.31 & 0.71 & 0.00 \\
\hline Reading normal size newsprint & 0.82 & 0.31 & 0.11 & 0.11 & 0.64 & -0.46 \\
\hline Distinguishing a person's features across the street & 0.81 & 0.22 & 0.19 & 0.24 & 0.64 & -0.96 \\
\hline
\end{tabular}

redundant and should be excluded from the average score for domain 1 .

Following the removal of these two items, Cronbach's alpha remained high at 0.95 .

\section{DISCUSSION}

Most quality of life instruments have to date been analysed and developed using classic test theory. ${ }^{4}$ More recently IRT ${ }^{7}$ has been recommended for assessing vision disability questionnaires. $^{8}$

We found that IRT ranked the items in the DLTV in terms of ease of performance. On dividing the questionnaire into two components on the basis of ease of doing, the 11 most difficult items are seen at the bottom of column $\mathrm{C}$ in table 2. Included in this list is "adjusting to darkness after being in the light." Although identified by IRT as being difficult, PCA recognised that the behaviour of this item was different. "Adjusting to light" was proposed by the focus group as an activity which caused difficulty. We decided to add "difficulty adjusting to darkness after being in the light" because dark adaptation may be abnormal in early AMD. ${ }^{9}$ We are intrigued that IRT rates this as more difficult than adjusting to light, and we will investigate responses to this item in future studies.

Internal consistency was highest in domain 1 at 0.96 . Generally speaking, a very high internal consistency suggests redundancy among items. Many would consider an alpha $>0.7$ to indicate good internal consistency and a value of $>0.9$ as indicating redundancy. ${ }^{6}$ We would argue that in a questionnaire that is addressing visual issues exclusively, a higher level of alpha would be acceptable and we note that other investigators have not rejected an alpha level of higher than 0.9 in other visual function questionnaires. ${ }^{4}{ }^{10} 11$

Some investigators identify redundancy based on the correlation of the item score to total score or by calculating Cronbach's alpha when each item is removed in turn, seeking to obtain their optimal level of alpha. ${ }^{4}$ We chose to exclude items when a significant amount of the variability in them could be explained by the remaining items. In domain 1 two items were excluded on this basis.

We have avoided naming the domains within the DLTV, as has been done previously in other questionnaires. ${ }^{10-13}$ Names often imply a clear understanding of the reason the different items group together within a domain-for example, "reading vision." However some other unrecognised common factor may cause items to segregate together. Domain names such as "visual expectations," "role problems,"10 or "miscellaneous information"'11 are based solely on the judgments of investigators. Therefore we chose to describe the domains in numerical groups (D1, D2, D3, and D4), in the order in which components emerged in PCA. The numerical assignment merely indicates the rank order of the contribution made to the amount of variance seen. D1 and D2 are strong domains, with high internal consistencies, and which discriminate between easily achieved items and more difficult ones. We would therefore expect Dl to be more sensitive to early loss in function. The items within D3 may all be related to "peripheral vision," but there may be some other aspect of visual function which links them together. D4 could be named "adjusting to light and dark," but it may be wiser to address the responses to these items individually, given its lower internal consistency.

In the present study, we have adopted a statistical approach to the allocation of items to domains. We did not think the allocation of items from PCA was counterintuitive and IRT provided some rationale for these allocations. In a previous study on the relations between visual function in older people and self reported ability to care for themselves or provide care to a dependant, we presented the DLTV findings using a preliminary domain structure ${ }^{14}$; however, the domain structure in the present study is the result of a more detailed and robust assessment. Nevertheless, there are only minor differences between the domain structure as previously described and that reported in the present study. The three items on global visual function that were scored differently from the rest were excluded from PCA in the present analysis. 
We think these should be analysed as individual items. PCA of the remaining 22 items yielded a domain structure which was almost identical to that previously reported. Only one item (from domain 3) was allocated to a different domain (domain 1). This item related to confidence in an unfamiliar neighbourhood and IRT identified this as a difficult item. Additionally, in the current domain structure we have taken into account redundancy of items and reduced domain 1 by two items. We now look forward to presenting data from the DLTV in this format in the future and invite other researchers to use it where they think appropriate.

\section{ACKNOWLEDGEMENTS}

This work was supported in part by a strategic project grant G9404234 from the Medical Research Council, UK and by a grant from the Guide Dogs for the Blind, UK.

\section{Authors' affiliations \\ P M Hart, A-M Montgomery, K A Muldrew, U Chakravarthy, Ophthalmology and Vision Science, The Queen's University of Belfast and Royal Group of Hospitals, Grosvenor Road, Belfast BT1 2 6BA, UK M R Stevenson, Clinical Research Support Centre, Royal Group Hospitals, Belfast BT12 6BA, UK \\ Competing interests: none declared}

Correspondence to: Patricia Hart, Department of Ophthalmology and Vision Science, The Queen's University of Belfast and Royal Group of Hospitals, Grosvenor Road, Belfast BT12 6BA, UK; pat.hart@ royalhospitals.n-i.nhs.uk

Accepted for publication 13 December 2004

\section{REFERENCES}

1 Hart PM, Chakravarthy U, Stevenson MR, et al. A vision specific functional index for use in patients with age related macular degeneration. Br J Ophthalmol 1999;83:1115-20.

2 McClure ME, Hart PM, Jackson AJ, et al. Macular degeneration: do conventional measurements of impaired visual function equate with visual disability? Br J Ophthalmol 2000;84:244-50.

3 Hart PM, Chakravarthy U, Mackenzie G, et al. Visual outcomes in the subfoveal radiotherapy study. Arch Ophthalmol 2002;120:1029-37.

4 Haymes SA, Johnston AW, Heyes AD. The development of the Melbourne low-vision ADL index: a measure of vision disability. Invest Ophthalmol Vis Sci 2001;42:1215-25.

5 McCullagh P. Regression models for ordinal data. J Roy Stat Soc, 1980;B, 42:109-42.

6 Massof RW, Rubin GS. Visual function assessment questionnaires. Surv Ophthalmol 2001;45:531-548.

7 Spector W, Fleishman J. Combining activities of daily living with instrumental activities of daily living to measure functional disability. J Gerontol B Psychol Sci Soc Svi 1998;53:S46-57.

8 Turano KA, Massof RW, Quigley HA. A self-assessment instrument designed for measuring independent mobility in RP patients: generalizability to glaucoma patients. Invest Ophthalmol Vis Sc 2002;43:2874-81.

9 Guymer RH, Gross-Jendroska M, Owens SL, et al. Laser treatment in subjects with high-risk clinical features of age-related macular degeneration. Posterior pole appearance and retinal function. Arch Ophthalmol 1997; 115:595-603.

10 Mangione CM, Phillips RS, Seddon JM, et al. Development of the Activities of Daily Vision Scale. Medical Care 1992;30:1111-26.

11 Frost NA, Sparrow JM, Durant JS, et al. Development of a questionnaire for measurement of vision-related quality of life. Ophthalmic Epidemiol 1998;5:185-210.

12 Mangione CM, Berry S, Spritzer K, et al. Identifying the content area for the 51-item National Eye Institute Visual Function Questionnaire: results from focus groups with visually impaired persons. Arch Ophthalmol 1998; 116:227-33.

13 Ware JE, Sherbourne CD. The MOS 36 item short form health survey (SF36). Conceptual framework and item selection. Medical Care 1992;30:473-83.

14 Stevenson MR, Hart PM, Montgomery AM, et al. Reduced vision in older adults with age-related macular degeneration interferes with ability to care for self and impairs role as carer. Br J Ophthalmol 2004;88:1125-30. 